\title{
Genuine deformations of submanifolds II: the conformal case
}

\author{
Luis A. Florit And Ruy TOJeIro
}

\begin{abstract}
We extend to the conformal realm the concept of genuine deformations of submanifolds, introduced by Dajczer and the first author for the isometric case. Analogously to that case, we call a conformal deformation of a submanifold $M^{n}$ genuine if no open subset of $M^{n}$ can be included as a submanifold of a higher dimensional conformally deformable submanifold in such a way that the conformal deformation of the former is induced by a conformal deformation of the latter. We describe the geometric structure of a submanifold that admits a genuine conformal deformation and give several applications showing the unifying character of this concept.
\end{abstract}

\section{Introduction}

An isometric immersion $\hat{f}: M^{n} \rightarrow \mathbb{R}^{n+q}$ with codimension $q$ of an $n$-dimensional Riemannian manifold $M^{n}$ into Euclidean space is said to be a genuine isometric deformation of a given isometric immersion $f: M^{n} \rightarrow \mathbb{R}^{n+p}$ if $f$ and $\hat{f}$ are nowhere (i.e., on no open subset of $M^{n}$ ) compositions, $f=F \circ j$ and $\hat{f}=\hat{F} \circ j$, of an isometric embedding $j: M^{n} \hookrightarrow N^{n+r}$ into a Riemannian manifold $N^{n+r}$ with $r>0$ and isometric immersions $F: N^{n+r} \rightarrow \mathbb{R}^{n+p}$ and $\hat{F}: N^{n+r} \rightarrow \mathbb{R}^{n+q}$ :

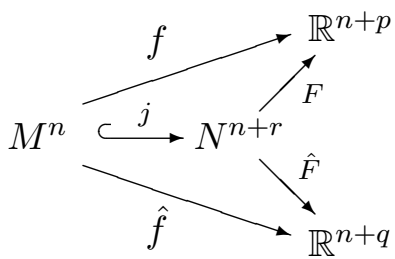

More geometrically, an isometric deformation of an Euclidean submanifold $M^{n}$ is genuine if no open subset of $M^{n}$ can be included as a submanifold of a higher dimensional isometrically deformable submanifold in such a way that the isometric deformation of the former is induced by an isometric deformation of the latter. 
This concept was introduced in [4], where it was proved that if an isometric immersion $f: M^{n} \rightarrow \mathbb{R}^{n+p}$ and a genuine isometric deformation $\hat{f}: M^{n} \rightarrow \mathbb{R}^{n+q}$ of it have sufficiently low codimensions then they are mutually (isometrically) ruled, that is, $M^{n}$ carries an integrable $d$-dimensional distribution $D^{d} \subset T M$ whose leaves are mapped diffeomorphically by $f$ and $\hat{f}$ onto open subsets of affine subspaces of $\mathbb{R}^{n+p}$ and $\mathbb{R}^{n+q}$, respectively. The authors also obtained a sharp estimate on the dimension $d$ of the rulings and proved that the normal connections and second fundamental forms of $f$ and $\hat{f}$ satisfy strong additional relations.

Besides containing several previous results on isometric deformations of submanifolds as particular cases, this concept has given new geometric insight on the structure of isometrically deformable submanifolds, showing that genuinely deformable submanifolds are rather special and providing an important step for extending to higher codimensions the classical SbranaCartan theory of isometrically deformable hypersurfaces $[1,9,13]$.

Our goal in this article is two-fold. First, to extend the notion of genuine deformations to the conformal realm, and to give a similar description as in [4] of the geometric nature of a submanifold that admits such a deformation. In particular, to provide a unified account of several known results on conformal deformations of submanifolds. Second, to understand geometrically the similitude between the theories of isometric and conformal deformations of submanifolds. In order to state our results we first set up some terminology.

A conformal structure on a manifold $M^{n}$ is an equivalence class of conformal Riemannian metrics on $M^{n}$. Recall that two Riemannian metrics $\langle$, and $\langle,\rangle^{\prime}$ on $M^{n}$ are conformal if there exists a positive smooth function $\varphi$ on $M^{n}$ such that $\langle,\rangle^{\prime}=\varphi^{2}\langle$,$\rangle . We call \varphi$ the conformal factor relating the metrics $\langle$,$\rangle and \langle,\rangle^{\prime}$. Clearly, every Riemannian manifold has a canonical conformal structure determined by its metric.

Given an immersion $f: M^{n} \rightarrow \bar{M}^{m}$ between differentiable manifolds, since conformal metrics on $\bar{M}^{m}$ are pulled-back by $f$ to conformal metrics on $M^{n}$, a conformal structure on $\bar{M}^{m}$ induces a conformal structure on $M^{n}$, the conformal structure on $M^{n}$ induced by $f$. If $M^{n}$ is already endowed with a conformal structure, we call $f$ conformal if such conformal structure coincides with that induced by $f$.

A pair $\{f, \bar{f}\}$ of conformal immersions $f: M^{n} \rightarrow \mathbb{R}^{n+p}$ and $\bar{f}: M^{n} \rightarrow$ $\mathbb{R}^{n+q}$ will be referred to simply as a conformal pair. We say that the conformal pair $\{f, \bar{f}\}$ extends conformally when there exists a conformal embed$\operatorname{ding} j: M^{n} \rightarrow N^{n+r}$, with $r \geq 1$, and conformal immersions $F: N^{n+r} \rightarrow$ $\mathbb{R}^{n+p}$ and $\bar{F}: N^{n+r} \rightarrow \mathbb{R}^{n+q}$ such that $f=F \circ j$ and $\bar{f}=\bar{F} \circ j$; see (1). We call the (ordered) conformal pair $\{F, \bar{F}\}$ a conformal extension of $\{f, \bar{f}\}$. 
The conformal pair $\{f, \bar{f}\}$ is said to be genuine if there is no open subset $U \subseteq M^{n}$ such that the restricted pair $\left\{\left.f\right|_{U},\left.\bar{f}\right|_{U}\right\}$ extends conformally. If $\{f, \bar{f}\}$ is a genuine conformal pair, we also say that each of its elements is a genuine conformal deformation of the other.

A conformal immersion $f: M^{n} \rightarrow \mathbb{R}^{n+p}$ is genuinely conformally rigid in $\mathbb{R}^{n+q}$ for a fixed integer $q>0$ if, for any given conformal immersion $\bar{f}: M^{n} \rightarrow \mathbb{R}^{n+q}$, there is an open dense subset $U \subset M^{n}$ such that the pair $\left\{\left.f\right|_{U},\left.\bar{f}\right|_{U}\right\}$ extends conformally.

Similar definitions can be given for any ambient spaces carrying conformal structures, as well as for isometric immersions between arbitrary semi-Riemannian manifolds, in the same way as in [4].

We say that an immersion $f: M^{n} \rightarrow \mathbb{R}^{n+p}$ is $D^{d}$-conformally ruled if $M^{n}$ carries an integrable $d$-dimensional distribution $D^{d} \subset T M$ whose leaves are mapped diffeomorphically by $f$ onto open subsets of affine subspaces or round spheres of $\mathbb{R}^{n+p}$. Then, at each point $x \in M^{n}$ we have a symmetric bilinear form $\beta^{f}=\beta^{f}(x): T_{x} M \times T_{x} M \rightarrow T_{x}^{\perp} M$ defined by

$$
\beta^{f}(Z, X):=\alpha^{f}(Z, X)-\langle Z, X\rangle \eta(x)
$$

and a subspace of the normal space $T_{x}^{\perp} M$ of $f$ at $x$ given by

$$
L_{D}^{\mathrm{c}}(x)=L_{D}^{\mathrm{c}}(f)(x)=\operatorname{span}\left\{\beta^{f}(Z, X): Z \in D^{d}(x) \quad \text { and } \quad X \in T_{x} M\right\} .
$$

Here $\alpha^{f}: T M \times T M \rightarrow T_{f}^{\perp} M$ stands for the second fundamental form of $f$ and $\eta(x)$ for the normal component of the mean curvature vector of the (image by $f$ of the) leaf of $D$ through $x \in M^{n}$. We always work on open subsets where the dimension of $L_{D}^{\mathrm{c}}(x)$ is constant, in which case such subspaces form a smooth subbundle of $T_{f}^{\perp} M$ that we denote by $L_{D}^{\mathrm{c}}$. Observe that, since $\beta^{f}(D, T M) \subseteq L_{D}^{\mathrm{c}}$, we have $D \subseteq \mathcal{N}\left(\beta_{L_{D}^{\mathrm{c} \perp}}^{f}\right)$, where $\mathcal{N}(\beta)$ denotes the nullity space of a symmetric bilinear form $\beta$, and a subspace as a subscript means to take the orthogonal projection onto that subspace.

We are now in a position to state the main result of this paper. As we will see, it implies or even generalizes main results in several other works, e.g., $[3,7,10-12]$.

Theorem 1.1. Let $f: M^{n} \rightarrow \mathbb{R}^{n+p}$ and $\bar{f}: M^{n} \rightarrow \mathbb{R}^{n+q}$ form a genuine conformal pair, with $p+q \leq n-3$ and $\min \{p, q\} \leq 5$. Then, along each connected component of an open dense subset of $M^{n}$, the immersions $f$ and $\bar{f}$ are mutually conformally $D^{d}$-ruled, with

$$
D^{d}=\mathcal{N}\left(\beta_{L^{\perp}}^{f}\right) \cap \mathcal{N}\left(\beta_{\bar{L}^{\perp}}^{\overline{\bar{l}}}\right) \quad \text { and } \quad d \geq n-p-q+3 \ell_{D}^{\mathrm{c}},
$$


where $L:=L_{D}^{\mathrm{c}}(f), \bar{L}:=L_{D}^{\mathrm{c}}(\bar{f})$ and $\ell_{D}^{\mathrm{c}}:=\operatorname{rank} L=\operatorname{rank} \bar{L}$. Moreover, there exists a parallel vector bundle isometry $\mathcal{T}: L \rightarrow \bar{L}$ such that $\beta_{\bar{L}}^{\bar{f}}=\varphi \mathcal{T} \circ \beta_{L}^{f}$, where $\varphi$ is the conformal factor relating the metrics induced by $f$ and $\bar{f}$.

In other words, up to an identification, the normal bundles of the immersions contain a subbundle $L=\bar{L}$ with the same normal connections and the same (conformal) second fundamental forms. On the other hand, the common conformal rulings $D^{d}$ of the immersions are the nullity of the (conformal) second fundamental forms on their orthogonal complements $L^{\perp}$ and $\bar{L}^{\perp}$. The larger is $L$, the bigger is $d$. We point out that Example 2 in [4] also shows that the estimate on $d$ in Theorem 1.1 is sharp.

As a consequence of Theorem 1.1, we obtain the following conformal version of the main result of [6]. We recall from [3] that the conformal $s$-nullity $\nu_{s}^{\mathrm{c}}(x)$ of an immersion $f: M^{n} \rightarrow \mathbb{R}^{n+p}$ at a point $x \in M^{n}$ is defined for $1 \leq s \leq p$ by

$$
\nu_{s}^{\mathrm{c}}(x)=\max \left\{\operatorname{dim} \mathcal{N}\left(\alpha_{V^{s}}^{f}(x)-\langle,\rangle_{f} \zeta\right): V^{s} \subset T_{x}^{\perp} M, \zeta \in V_{s}\right\}
$$

where $\langle,\rangle_{f}$ stands for the metric on $M^{n}$ induced by $f$.

Corollary 1.1. Let $f: M^{n} \rightarrow \mathbb{R}^{n+p}$ be an immersion and let $q$ be a positive integer with $p \leq q \leq n-p-3$. Suppose that $p \leq 5$ and that $f$ satisfies

$$
\nu_{s}^{\mathrm{c}} \leq n+p-q-2 s-1 \quad \text { for all } \quad 1 \leq s \leq p .
$$

For $q \geq p+5$ assume further that $\nu_{1}^{\mathrm{c}} \leq n-2(q-p)+1$. Then, any immersion $\bar{f}: M^{n} \rightarrow \mathbb{R}^{n+q}$ conformal to $f$ is locally a composition, i.e., there exists an open dense subset $V \subseteq M^{n}$ such that the restriction $\bar{f}$ to any connected component $U$ of $V$ satisfies $\left.\bar{f}\right|_{U}=\left.h \circ f\right|_{U}$, where $h: W \subset \mathbb{R}^{n+p} \rightarrow \mathbb{R}^{n+q}$ is a conformal immersion of an open subset $W \supset f(U)$.

For $p=q$, the preceding corollary extends up to codimension $p=5$ the main theorem of [3], which ensures conformal rigidity of $f$ in $\mathbb{R}^{n+p}$ whenever $p \leq 4$ and $\nu_{s}^{\mathrm{c}} \leq n-2 s-1$ for all $1 \leq s \leq p$. The latter, in its turn, is a generalization of Cartan's classical criterion $\nu_{s}^{\mathrm{c}} \leq n-2$ for conformal rigidity of hypersurfaces. Corollary 1.1 also generalizes the main result of [11], which deals with the special case $p=1$, as well as [14] up to codimension 5 .

If we apply Theorem 1.1 for $p=q=2$, the estimate on $d$ implies that $\ell_{D}^{\mathrm{c}} \leq 1$. This yields $d \geq n-4$ if $\ell_{D}^{\mathrm{c}}=0$ and $d \geq n-1$ if $\ell_{D}^{\mathrm{c}}=1$. In both cases, the conformal nullity $\nu^{\mathrm{c}}=\nu_{2}^{\mathrm{c}}$ of both immersions satisfies $\nu^{\mathrm{c}} \geq n-4$. 
Therefore, under the assumptions that $n \geq 7$ and $\nu_{f}^{\mathrm{c}} \leq n-5$ everywhere, we conclude that $f$ is genuinely conformally rigid. Here, this means that if $\bar{f}$ is a conformal deformation of $f$, then there exists an open and dense subset of $M^{n}$ on each connected component of which either $\bar{f}$ is conformally congruent to $f$ or $f$ can be included as a submanifold of either a conformally flat or a Cartan hypersurface and $\bar{f}$ is induced by a conformal deformation of such hypersurface. This is the content of the main theorem in [12].

Clearly, Theorem 1.1 yields the following criterion for genuine conformal rigidity.

Corollary 1.2. Let $f: M^{n} \rightarrow \mathbb{R}^{n+p}$ be a conformal immersion and let $q$ be a positive integer with $p+q \leq n-3$ and $\min \{p, q\} \leq 5$. If $f$ is not $(n-p-q)$-conformally ruled on any open subset of $M^{n}$, then $f$ is genuinely conformally rigid in $\mathbb{R}^{n+q}$.

Our next result gives a geometric way to construct genuine conformal pairs by means of isometric ones, explaining the similarity between Theorem 1.1 and its isometric counterpart in [4]. To do this, we need to introduce some further terminology.

Let $\mathbb{L}^{N+2}$ denote the $(N+2)$-dimensional Lorentz space, and let

$$
\mathbb{V}^{N+1}=\left\{x \in \mathbb{L}^{N+2}:\langle x, x\rangle=0\right\}
$$

be the light cone in $\mathbb{L}^{N+2}$. Fix a pseudo-orthonormal basis $\left\{e_{0}, e_{1}, \ldots, e_{N+1}\right\}$ of $\mathbb{L}^{N+2}$, that is,

$$
\left\langle e_{0}, e_{0}\right\rangle=\left\langle e_{1}, e_{1}\right\rangle=0,\left\langle e_{0}, e_{1}\right\rangle=1
$$

and $\left\{e_{2}, \ldots, e_{N+1}\right\}$ is an orthonormal basis of the Riemannian subspace $\left\{e_{0}, e_{1}\right\}^{\perp}$. Then

$$
\mathbb{E}^{N}=\left\{x \in \mathbb{V}^{N+1}:\left\langle x, e_{0}\right\rangle=1\right\}
$$

is a model of $N$-dimensional Euclidean space: the map $\Psi: \mathbb{R}^{N} \rightarrow \mathbb{L}^{N+2}$ defined by

$$
\Psi(x)=-\frac{\|x\|^{2}}{2} e_{0}+e_{1}+\sum_{i=1}^{N} x_{i} e_{i+1}
$$

is an isometric embedding with $\Psi\left(\mathbb{R}^{N}\right)=\mathbb{E}^{N}$.

Given an immersion $g: M^{n} \rightarrow \mathbb{V}^{N+1}$ of a differentiable manifold $M^{n}$, for any positive $\mu \in C^{\infty}\left(M^{n}\right)$ the map $h: M^{n} \rightarrow \mathbb{V}^{N+1}$ given by $h=\mu g$ 
is also an immersion, and the induced metrics $\langle,\rangle_{g}$ and $\langle,\rangle_{h}$ are related by $\langle,\rangle_{h}=\mu^{2}\langle,\rangle_{g}$. Therefore, if $\langle,\rangle_{g}=\varphi^{2}\langle$,$\rangle for some fixed metric \langle$,$\rangle on$ $M^{n}$, then $h$ can be made isometric with respect to $\langle$,$\rangle by choosing \mu=\varphi^{-1}$. In particular, if $g=\Psi \circ f$ for some conformal immersion $f: M^{n} \rightarrow \mathbb{R}^{N}$ of a Riemannian manifold, then such an $h$ is denoted by $\mathcal{I}(f)$ and called the isometric light cone representative of $f$.

On the other hand, if $g: M^{n} \rightarrow \mathbb{V}^{N+1}$ is such that $g\left(M^{n}\right) \subset \mathbb{V}^{N+1} \backslash \mathbb{R}_{e_{0}}$, where $\mathbb{R}_{e_{0}}=\left\{t e_{0}: t>0\right\}$, define $\mathcal{C}(g): M^{n} \rightarrow \mathbb{R}^{N}$ by $\Psi \circ \mathcal{C}(g)=\left\langle g, e_{0}\right\rangle^{-1} g$. Since $\Psi$ is an isometric immersion, it follows that $g$ and $\mathcal{C}(g)$ induce conformal metrics on $M^{n}$ with conformal factor $\left\langle g, e_{0}\right\rangle^{-1}$. Clearly, $g=\mathcal{I}(f)$ if $f=\mathcal{C}(g)$ for an isometric immersion $g: M^{n} \rightarrow \mathbb{V}^{N+1}$ of a Riemannian manifold.

This leads to the following procedure to construct a conformal pair of immersions $f: M^{n} \rightarrow \mathbb{R}^{n+p}$ and $\bar{f}: M^{n} \rightarrow \mathbb{R}^{n+q}$ : start with a Riemannian manifold $N^{n+1}$ that admits an isometric immersion $F^{\prime}: N^{n+1} \rightarrow \mathbb{R}^{n+p}$ and an isometric embedding $\hat{F}: N^{n+1} \rightarrow \mathbb{L}^{n+q+2}$ transversal to the light cone $\mathbb{V}^{n+q+1}$. Then set $M^{n}:=\hat{F}^{-1}\left(\hat{F}\left(N^{n+1}\right) \cap \mathbb{V}^{n+q+2}\right), f=F^{\prime} \circ i$ and $\bar{f}=$ $\mathcal{C}(\hat{F} \circ i)$, where $i: M^{n} \rightarrow N^{n+1}$ is the inclusion map.

The following result states that any genuine conformal pair $\{f, \bar{f}\}$ of Euclidean submanifolds in sufficiently low codimensions is locally produced in this way from a genuine isometric pair $\{F, \hat{F}\}$ as above.

Theorem 1.2. Assume that $f: M^{n} \rightarrow \mathbb{R}^{n+p}, p \geq 1$, and $\bar{f}: M^{n} \rightarrow \mathbb{R}^{n+q}$ form a genuine conformal pair, with $p+q \leq n-3$ and $\min \{p, q\} \leq 5$. Suppose further that $\bar{f}$ is nowhere conformally congruent to an immersion that is isometric to $f$. Then (locally on an open dense subset of $M^{n}$ ) there exist a Riemannian manifold $N^{n+1}$ that admits an isometric immersion $F^{\prime}: N^{n+1} \rightarrow \mathbb{R}^{n+p}$ and an isometric embedding $\hat{F}: N^{n+1} \rightarrow \mathbb{L}^{n+q+2}$ transversal to the light cone $\mathbb{V}^{n+q+1}$, and a conformal diffeomorphism $i: M^{n} \rightarrow$ $\hat{F}^{-1}\left(\hat{F}\left(N^{n+1}\right) \cap \mathbb{V}^{n+q+1}\right)$ such that $\left\{F^{\prime}, \hat{F}\right\}$ is a genuine isometric pair, $f=$ $F^{\prime} \circ i$ and $\bar{f}=\mathcal{C}(\hat{F} \circ i)$.

Note that the assumption that $\bar{f}$ is nowhere locally conformally congruent to an immersion that is isometric to $f$ is always satisfied if $f$ is genuinely isometrically rigid in $\mathbb{R}^{n+q}$, for instance if $M^{n}$ does not carry any ruled open subset with rulings of dimension at least $n-p-q$. In particular, this is always the case after composing $f$ with a suitable inversion of $\mathbb{R}^{n+p}$.

For $p=1$, Theorem 1.2 says that any hypersurface $f: M^{n} \rightarrow \mathbb{R}^{n+1}$ that admits a genuine conformal (but not isometric) deformation in $\mathbb{R}^{n+q}, q \leq$ 
$n-4$, can be locally produced as the intersection of an $(n+1)$-dimensional flat submanifold of $\mathbb{L}^{n+q+2}$ with the light cone:

Corollary 1.3. Let $f: M^{n} \rightarrow \mathbb{R}^{n+1}$ and $\bar{f}: M^{n} \rightarrow \mathbb{R}^{n+q}$ form a conformal pair, with $q \leq n-4$. Assume that there exists no open subset $M^{n}$ along which $\bar{f}$ is either a composition or it is conformally congruent to an isometric deformation of $f$. Then, (locally on an open dense subset of $M^{n}$ ) there exist an isometric embedding $\bar{F}: U \subset \mathbb{R}^{n+1} \rightarrow \mathbb{L}^{n+q+2}$ transversal to the light cone $\mathbb{V}^{n+q+1}$ and a conformal diffeomorphism $\tau: M^{n} \rightarrow \bar{M}^{n}:=$ $\bar{F}^{-1}\left(\bar{F}(U) \cap \mathbb{V}^{n+q+1}\right) \subset U$ such that $f=i \circ \tau$ and $\bar{f}=\mathcal{C}(\bar{F} \circ \tau)$, where $i: \bar{M}^{n} \rightarrow U$ is the inclusion map.

In the particular case $q=1$, the above reduces to Theorem 1 in [10], which can be regarded as a nonparametric description of Cartan's conformally deformable hypersurfaces.

Another important special case of Theorem 1.2 occurs when $q=0$. In this situation, we consider a conformally flat submanifold $f: M^{n} \rightarrow \mathbb{R}^{n+p}$, which clearly forms a genuine conformal pair with any conformal diffeomorphism $\bar{f}: M^{n} \rightarrow U \subset \mathbb{R}^{n}$ onto an open subset. Then we recover Theorem 1 from [7], which gives a geometric procedure to construct all conformally flat Euclidean submanifolds in low codimension:

Corollary 1.4. Let $f: M^{n} \rightarrow \mathbb{R}^{n+p}, n \geq 5, p \leq n-3$, be a conformal immersion of a conformally flat manifold. Assume that the metric induced by $f$ is nowhere flat. Then (locally on an open dense subset) there exist a Riemannian manifold $N^{n+1}$ that admits an isometric immersion $F: N^{n+1} \rightarrow$ $\mathbb{R}^{n+p}$ and an isometric embedding $\bar{F}: N^{n+1} \rightarrow \mathbb{L}^{n+2}$, and a conformal diffeomorphism $\tau: M^{n} \rightarrow \bar{M}^{n}:=\bar{F}^{-1}\left(\bar{F}\left(N^{n+1}\right) \cap \mathbb{V}^{n+1}\right)$ such that $f=F \circ \tau$.

Our approach to study the geometric structure of a conformal pair of immersions $f: M^{n} \rightarrow \mathbb{R}^{n+p}$ and $\bar{f}: M^{n} \rightarrow \mathbb{R}^{n+q}$ is, as usual, to fix on $M^{n}$ the Riemannian metric induced by one of the immersions, say, $f$, and to reduce the problem to the study of the isometric pair of immersions $\{f, \hat{f}\}$ that arises by considering the isometric light cone representative $\hat{f}$ of $\bar{f}$. In contrast to the theory developed in [4], there are two cases to consider. The first (nondegenerate) case can be treated in a similar way as in [4]. However, in the second one it appears a certain degeneracy condition that requires a more delicate approach involving the light cone representatives of both $f$ and $\hat{f}$. Therefore, as a first and main step, in the next section we extend the theory developed in [4] to isometric pairs of immersions into flat semi-Riemannian spaces. 
Before we conclude this introduction, one final remark is in order. Although we deal only with pairs of immersions in this paper, when studying some rigidity phenomena of submanifolds one is naturally led to consider sets of immersions, not only pairs. For instance, it was shown in Theorem 5 in [5] that the associated family $\left\{f_{\theta}: M^{2 n} \rightarrow \mathbb{R}^{2 n+2}: \theta \in[0, \pi)\right\}$ of a minimal nonholomorphic Kahler submanifold $f_{0}$ of rank two in codimension two does not extend isometrically, although for any $\theta_{1} \neq \theta_{2} \in[0, \pi)$ the pair $\left\{f_{\theta_{1}}, f_{\theta_{2}}\right\}$ does extend. Here, we say that a set $\left\{f_{i}: M^{n} \rightarrow N_{i}^{n+p_{i}}: i \in I\right\}$ of isometric (resp., conformal) immersions indexed by an arbitrary set $I$ extends isometrically (resp., conformally), when there exist an isometric (resp., conformal) embedding $j: M^{n} \rightarrow N^{m}$, with $m>n$, and a set $\left\{F_{i}: N^{m} \rightarrow N_{i}^{n+p_{i}}: i \in I\right\}$ of isometric (resp., conformal) immersions such that $f_{i}=F_{i} \circ j$, for all $i \in I$.

\section{Isometric pairs into flat semi-Riemannian spaces}

In this section, we study the structure of the tangent and normal bundles of a pair of isometric immersions into flat semi-Riemannian spaces. Our goal is to give conditions that allow the construction of isometric ruled extensions.

\subsection{Semi-Riemannian ruled isometric extensions}

In this subsection, we give general conditions for the existence of isometric ruled extensions of a pair of isometric immersions into flat semi-Riemannian spaces. The proofs are identical to the ones for the Riemannian case [4] and will be omitted.

Throughout the paper, given a bilinear form $\beta: V^{n} \times U^{m} \rightarrow W$ between finite-dimensional real vector spaces, we denote by $\mathcal{S}(\beta) \subset W$ the subspace spanned by the image of $\beta$, that is,

$$
\mathcal{S}(\beta)=\operatorname{span}\left\{\beta(X, Y): X \in V^{n} \text { and } Y \in U^{m}\right\}
$$

and by $\mathcal{N}(\beta) \subset V^{n}$ the (left) nullity space of $\beta$ defined as

$$
\mathcal{N}(\beta)=\left\{X \in V^{n}: \beta(X, Y)=0 \text { for all } Y \in U^{m}\right\} .
$$

If $W$ is endowed with a nondegenerate inner product $\langle$,$\rangle and T \subseteq W$ is a nondegenerate subspace with respect to $\langle$,$\rangle , we denote \beta_{T}=\pi_{T} \circ \beta$, where $\pi_{T}$ is the orthogonal projection onto $T$. Then

$$
\mathcal{N}\left(\beta_{T}\right)=\left\{X \in V^{n}:\langle\beta(X, Y), \xi\rangle=0 \text { for all } Y \in U^{m}, \xi \in T\right\}
$$


We also denote by $\mathcal{N}\left(\beta_{T}\right)$ the subspace defined as above even if $T$ is degenerate.

Let $\mathbb{R}_{a}^{m}$ stand for $\mathbb{R}^{m}$ with the standard flat semi-Riemannian metric of index $a$. In particular, $\mathbb{R}_{1}^{m}=\mathbb{L}^{m}$. Let $f: M^{n} \rightarrow \mathbb{R}_{a}^{n+p}$ and $\hat{f}: M^{n} \rightarrow \mathbb{R}_{b}^{n+q}$ form an isometric pair. Assume that there exists a vector bundle isometry

$$
\mathcal{T}: L \subset T_{f}^{\perp} M \rightarrow \hat{L} \subset T_{\hat{f}}^{\perp} M
$$

between nondegenerate subbundles such that

$$
D=\mathcal{N}\left(\alpha_{L^{\perp}}\right) \cap \mathcal{N}\left(\hat{\alpha}_{\hat{L}^{\perp}}\right) \subset T M
$$

defines a smooth subbundle of $T M$ and such that the pair $(\mathcal{T}, D)$ satisfies the following two conditions:

$$
\left\{\begin{array}{l}
\left(\mathrm{C}_{1}\right) \text { The isometry } \mathcal{T} \text { is parallel and preserves second } \\
\quad \text { fundamental forms, } \\
\left(\mathrm{C}_{2}\right) \text { The subbundles } L \text { and } \hat{L} \text { are parallel along } D \\
\text { in the normal connections. }
\end{array}\right.
$$

Let $\phi:(T M \oplus L) \times T M \rightarrow L^{\perp} \times \hat{L}^{\perp}$ be the bilinear form given by

$$
\phi(Y+\xi, X)=\left(\left(\widetilde{\nabla}_{X}(Y+\xi)\right)_{L^{\perp}},\left(\widetilde{\nabla}_{X}(Y+\mathcal{T} \xi)\right)_{\hat{L}^{\perp}}\right),
$$

where $\widetilde{\nabla}$ stands for the connections of both $\mathbb{R}_{a}^{n+p}$ and $\mathbb{R}_{b}^{n+q}$, and assume further that the vector subspaces

$$
\Delta:=\mathcal{N}(\phi) \subset T M \oplus L
$$

have constant dimension on $M^{n}$. By condition $\left(\mathrm{C}_{1}\right)$, the vector bundle isometry defined as $T_{0}=I \oplus \mathcal{T}: f_{*} T M \oplus L \rightarrow \hat{f}_{*} T M \oplus \hat{L}$ is parallel with respect to the connections induced by the Euclidean ambient spaces. It follows that $\left.T_{0}\right|_{\Delta}: \Delta \rightarrow \hat{\Delta}$ is a parallel vector bundle isometry, and hence, we may identify $\hat{\Delta}$ with $\Delta$.

Lemma 2.1 [4]. The distribution $D \subset \Delta$ is integrable and $\Delta \cap T M=D$ holds.

Consider the vector bundle $\pi: \Lambda=\hat{\Lambda} \rightarrow M^{n}$ determined by the orthogonal splitting $\Delta=D \oplus \Lambda$, and define $F^{\prime}: N \rightarrow \mathbb{R}_{a}^{n+p}$ as the restriction of 
the map

$$
\lambda \in \Lambda \mapsto f(\pi(\lambda))+\lambda
$$

to a tubular neighborhood $N$ of the 0-section $j: M^{n} \hookrightarrow N \subset \Lambda$ of $\Lambda$ along which $F$ is an immersion. Similarly, define $\hat{F}: N \rightarrow \mathbb{R}_{b}^{n+q}$. Henceforth, $L^{\perp}$ and $\Delta$ will be understood as vector bundles over $N \subset \Lambda$ by means of $L^{\perp}(\lambda)=$ $L^{\perp}(\pi(\lambda))$ and $\Delta(\lambda)=\Delta(\pi(\lambda))$.

Proposition 2.1 [4]. The immersions $F^{\prime}$ and $\hat{F}$ are isometric $\Delta$-ruled extensions of $f$ and $\hat{f}$. Moreover, there are smooth orthogonal splittings

$$
T_{F^{\prime}}^{\perp} N=\mathcal{L} \oplus L^{\perp} \quad \text { and } \quad T_{\hat{F}}^{\perp} N=\hat{\mathcal{L}} \oplus \hat{L}^{\perp}
$$

and a vector bundle isometry $T: \mathcal{L} \rightarrow \hat{\mathcal{L}}$ such that

$$
\Delta=\mathcal{N}\left(\alpha_{\mathcal{L}^{\perp}}^{F^{\prime}}\right) \cap \mathcal{N}\left(\hat{\alpha}_{\hat{\mathcal{L}}^{\perp}}^{\hat{F}}\right)
$$

and the pair $(T, \Delta)$ satisfies conditions $\left(\mathrm{C}_{1}\right)$ and $\left(\mathrm{C}_{2}\right)$ in $(2.1)$.

Observe that if the ruled extensions $F^{\prime}$ and $\hat{F}$ are trivial (i.e., $\operatorname{dim} N=n$ ) then $f$ and $\hat{f}$ are themselves $D$-ruled.

\subsection{Construction of the pair $\left(\mathcal{T}, D^{d}\right)$ and the estimate on $d$}

In this subsection we show how to construct a pair $\left(\mathcal{T}, D^{d}\right)$ satisfying conditions $\left(\mathrm{C}_{1}\right)$ and $\left(\mathrm{C}_{2}\right)$ in (2.1) for a pair of isometric immersions $f: M^{n} \rightarrow$ $\mathbb{R}_{a}^{n+p}$ and $\hat{f}: M^{n} \rightarrow \mathbb{R}_{b}^{n+q}$, and we obtain an estimate on $d$. We follow closely the strategy in [4] for the case $a=0=b$. Here, however, two distinct cases arise, depending on whether a certain nondegeneracy condition is satisfied or not. The degenerate case requires several modifications in the arguments of [4], which will be carried out in Section 2.2.2 only in the case that is needed for our study of conformal pairs of immersions into Euclidean space.

Given an isometric pair of immersions $f: M^{n} \rightarrow \mathbb{R}_{a}^{n+p}$ and $\hat{f}: M^{n} \rightarrow$ $\mathbb{R}_{b}^{n+q}$, denote by $\alpha$ and $\hat{\alpha}$ their respective second fundamental forms and endow the vector bundle $T_{f}^{\perp} M \oplus T_{\hat{f}}^{\perp} M$ with the indefinite metric of type $(p, q)$ given by

$$
\langle\langle,\rangle\rangle_{T_{f}^{\perp} M \oplus T_{\hat{f}}^{\perp} M}=\langle,\rangle_{T_{f}^{\perp} M}-\langle,\rangle_{T_{\hat{f}}^{\perp} M}
$$

Set $\alpha \oplus \hat{\alpha}: T M \times T M \rightarrow \mathcal{S}(\alpha) \oplus \mathcal{S}(\hat{\alpha}) \subset T_{f}^{\perp} M \oplus T_{\hat{f}}^{\perp} M$. 
Definition 2.1. We say that the pair $\{f, \hat{f}\}$ as above is nondegenerate if the projections of $\Omega=\Omega(f, \hat{f}):=\mathcal{S}(\alpha \oplus \hat{\alpha}) \cap \mathcal{S}(\alpha \oplus \hat{\alpha})^{\perp} \subset \mathcal{S}(\alpha) \oplus \mathcal{S}(\hat{\alpha})$ onto $T_{f}^{\perp} M$ and $T_{\hat{f}}^{\perp} M$ are injective. When this condition is nowhere satisfied, we say that the pair is degenerate.

Note that $\{f, \hat{f}\}$ is nondegenerate if both $\mathcal{S}(\alpha)$ and $\mathcal{S}(\hat{\alpha})$ are nondegenerate. In particular, this is the case if $a, b$ and the index of $M^{n}$ are all equal.

2.2.1. The nondegenerate case Assuming $\{f, \hat{f}\}$ to be nondegenerate, we have orthogonal splittings

$$
\mathcal{S}(\alpha)=\Gamma \oplus \Gamma^{\perp} \quad \text { and } \quad \mathcal{S}(\hat{\alpha})=\hat{\Gamma} \oplus \hat{\Gamma}^{\perp},
$$

where $\Gamma=\mathcal{S}(\alpha) \cap \Omega^{\perp}$ and $\hat{\Gamma}=\mathcal{S}(\hat{\alpha}) \cap \Omega^{\perp}$, and an isometry $\mathcal{J}: \Gamma^{\perp} \rightarrow \hat{\Gamma}^{\perp}$ such that

$$
\Omega=\left\{(\eta, \mathcal{J} \eta): \eta \in \Gamma^{\perp}\right\} \subset \Gamma^{\perp} \oplus \hat{\Gamma}^{\perp}
$$

and $\hat{\alpha}_{\hat{\Gamma}^{\perp}}=\mathcal{J} \circ \alpha_{\Gamma^{\perp}}$. From now on we identify $\Gamma^{\perp}$ with $\hat{\Gamma}^{\perp}$ by means of $\mathcal{J}$, and hence

$$
\hat{\alpha}_{\hat{\Gamma}^{\perp}}=\alpha_{\Gamma^{\perp}}
$$

Define $\beta: T M \times T M \rightarrow \Gamma \oplus \hat{\Gamma}$ as $\beta=\alpha_{\Gamma} \oplus \hat{\alpha}_{\hat{\Gamma}}$, and a vector subbundle $\Theta \subset T M$ by

$$
\Theta=\mathcal{N}(\beta) .
$$

The vector subbundle $S \subset \Gamma^{\perp}\left(=\hat{\Gamma}^{\perp}\right)$ defined by

$$
S=\mathcal{S}\left(\left.\alpha\right|_{\Theta \times T M}\right)
$$

satisfies $\Theta=\mathcal{N}\left(\alpha_{S^{\perp}}\right) \cap \mathcal{N}\left(\hat{\alpha}_{\hat{S}^{\perp}}\right)$. Now, given $X \in T M$, denote by $\mathcal{K}(X) \in$ $\Lambda^{2}(S)$ the skew-symmetric tensor given by

$$
\mathcal{K}(X) \eta=\left(\nabla \frac{\perp}{X} \eta\right)_{S}-\left(\hat{\nabla}_{X}^{\perp} \eta\right)_{\hat{S}},
$$

and define a vector subbundle $S_{0} \subset S$ by

$$
S_{0}=\bigcap_{X \in T M} \operatorname{ker} \mathcal{K}(X) .
$$

Then, define vector subbundles $L^{\ell} \subset S_{0}$ and $D^{d} \subset \Theta$ as

$$
L^{\ell}=\left\{\delta \in S_{0}: \nabla_{Y}^{\perp} \delta \in S \quad \text { and } \quad \hat{\nabla}_{Y}^{\perp} \delta \in \hat{S} \quad \text { for all } Y \in \Theta\right\}
$$


and

$$
D^{d}=\mathcal{N}\left(\alpha_{L^{\perp}}\right) \cap \mathcal{N}\left(\hat{\alpha}_{\hat{L}^{\perp}}\right),
$$

and let $\mathcal{T}: L^{\ell} \rightarrow L^{\ell}$ be the induced vector bundle isometry given by

$$
\mathcal{T}=\left.\mathcal{J}\right|_{L}: L^{\ell} \subset T_{f}^{\perp} M \rightarrow L^{\ell} \subset T_{\hat{f}}^{\perp} M
$$

With these definitions, we have the following:

Theorem 2.1 [4]. Let $f: M^{n} \rightarrow \mathbb{R}_{a}^{n+p}$ and $\hat{f}: M^{n} \rightarrow \mathbb{R}_{b}^{n+q}$ form a nondegenerate isometric pair of immersions of a semi-Riemannian manifold with index $\min \{a, b\}$. Then, along each connected component of an open dense subset of $M^{n}$, the pair $\left(\mathcal{T}, D^{d}\right)$ satisfies $\left(\mathrm{C}_{1}\right)$ and $\left(\mathrm{C}_{2}\right)$ in $(2.1)$. In particular, $f$ and $\hat{f}$ have (possibly trivial) maximal isometric $\Delta^{d+r}$-ruled extensions $F^{\prime}: N^{n+r} \rightarrow \mathbb{R}_{a}^{n+p}$ and $\hat{F}: N^{n+r} \rightarrow \mathbb{R}_{b}^{n+q}, 0 \leq r \leq \ell$, which form a nondegenerate pair and satisfy the conclusions of Proposition 2.1.

Moreover, if $p+q \leq n-1$ and $\min \{p+b-a, q+a-b\} \leq 6$ then

$$
d+r \geq n-p-q+3 \ell
$$

unless $\min \{p+b-a, q+a-b\}=6$ and $\ell=0$ in which case $d+r \geq n-$ $p-q+3 \ell-1$.

Remark 2.1. (i) The hypothesis on the codimensions in Theorem 2.1 is required in a fundamental algebraic result needed in its proof, whose most general version is Theorem 3 in [6]. Unfortunately, this algebraic result is false without that assumption [8].

(ii) We can relax the hypothesis on the index of $M$ by asking $S$ in (2.7) to be Riemannian.

The proof of the above result follows exactly as those of Theorems 11 and 14 in [4], where of course no hypothesis on the nondegeneracy was needed since both normal spaces are Riemannian when both ambient spaces are Euclidean. The only extra property to verify is that the pair $\left\{F^{\prime}, \hat{F}\right\}$ in Theorem 2.1 is nondegenerate, but this is immediate from Proposition 2.1, since $\alpha_{L}^{f}=\alpha_{\hat{L}}^{\hat{f}}, \alpha_{\mathcal{L}^{\prime}}^{F^{\prime}}=\hat{\alpha} \hat{\mathcal{L}}_{\hat{\mathcal{L}}}^{\hat{F}},\left.\alpha_{\mathcal{L}^{\perp}}^{F^{\prime}}\right|_{T M \times T M}=\alpha_{L^{\perp}}^{f},\left.\alpha_{\hat{\mathcal{L}}^{\perp}}^{\hat{F}}\right|_{T M \times T M}=\alpha_{\hat{L}^{\perp}}^{\hat{f}}$ and $\Delta=$ $\mathcal{N}\left(\alpha_{\mathcal{L}^{\perp}}^{F^{\prime}}\right) \cap \mathcal{N}\left(\hat{\alpha}_{\hat{\mathcal{L}}^{\perp}}^{\hat{F}}\right)$

2.2.2. The degenerate case In this subsection we address the degenerate case in the setting that will be needed for the next section, namely, 
for a pair of isometric immersions $f: M^{n} \rightarrow \mathbb{R}^{n+p}$ and $\hat{f}: M^{n} \rightarrow \mathbb{V}^{n+q+1} \subset$ $\mathbb{L}^{n+q+2}$ of a Riemannian manifold, under the assumptions that

$$
p+q \leq n-1 \quad \text { and } \min \{p, q\} \leq 5 .
$$

The main difficulty here is that the subbundle $\Gamma^{\perp}$, constructed in the preceding subsection for the nondegenerate case, is no longer well defined. To deal with this issue, we need to consider also the isometric representative of $f$ into the light cone.

Thus, here we assume that there is a null vector field $0 \neq \xi_{0} \in \mathcal{S}(\hat{\alpha}) \cap$ $\mathcal{S}(\hat{\alpha})^{\perp}$ such that $\left(0, \xi_{0}\right) \in \mathcal{S}(\alpha \oplus \hat{\alpha}) \cap \mathcal{S}(\alpha \oplus \hat{\alpha})^{\perp}$. Set $f^{\prime}=\mathcal{I}(f): M^{n} \rightarrow$ $\mathbb{V}^{n+p+1} \subset \mathbb{L}^{n+p+2}$ and denote by $\alpha^{\prime}$ its second fundamental form. Then, the position vector fields of both $f^{\prime}$ and $\hat{f}$ are normal, and

$$
A_{f^{\prime}}^{f^{\prime}}=A_{\hat{f}}^{\hat{f}}=-I, \quad A_{e_{0}}^{f^{\prime}}=A_{\xi_{0}}^{\hat{f}}=0 .
$$

Observe that $\left(f^{\prime}, \hat{f}\right) \in \mathcal{S}\left(\alpha^{\prime} \oplus \hat{\alpha}\right)^{\perp}$, but $\left(f^{\prime}, \hat{f}\right) \notin \mathcal{S}\left(\alpha^{\prime} \oplus \hat{\alpha}\right)$. Since the normal spaces of $\hat{f}$ have index 1 , we can assume further that $\left\langle\hat{f}, \xi_{0}\right\rangle=1$.

We will make a similar construction as in the nondegenerate case, but now for the pair $\left\{f^{\prime}, \hat{f}\right\}$. The idea is to force the inclusion of the vector $\left(f^{\prime}, \hat{f}\right)$ in $\Omega$, despite the fact that $\left(f^{\prime}, \hat{f}\right) \notin \mathcal{S}\left(\alpha^{\prime} \oplus \hat{\alpha}\right)$. We then define $\Omega \subset$ $\operatorname{span}\left\{\left(f^{\prime}, \hat{f}\right)\right\} \oplus \mathcal{S}\left(\alpha^{\prime}\right) \oplus \mathcal{S}(\hat{\alpha})$ as the vector bundle with null fibers

$$
\Omega:=\operatorname{span}\left\{\left(f^{\prime}, \hat{f}\right)\right\} \oplus\left(\mathcal{S}\left(\alpha^{\prime} \oplus \hat{\alpha}\right) \cap \mathcal{S}\left(\alpha^{\prime} \oplus \hat{\alpha}\right)^{\perp}\right) .
$$

Note that, by (2.11) and the definition of $\xi_{0}$, we get that $\left(e_{0}, \xi_{0}\right) \in \Omega$. As before, there are orthogonal splittings

$$
\operatorname{span}\left\{f^{\prime}\right\} \oplus \mathcal{S}\left(\alpha^{\prime}\right)=\Gamma \oplus \Gamma^{\perp} \quad \text { and } \quad \operatorname{span}\{\hat{f}\} \oplus \mathcal{S}(\hat{\alpha})=\hat{\Gamma} \oplus \hat{\Gamma}^{\perp},
$$

where $\Gamma=\mathcal{S}\left(\alpha^{\prime}\right) \cap \Omega^{\perp} \subseteq \operatorname{span}\left\{f^{\prime}, e_{0}\right\}^{\perp}$ and $\hat{\Gamma}=\mathcal{S}(\hat{\alpha}) \cap \Omega^{\perp} \subseteq \operatorname{span}\left\{\hat{f}, \xi_{0}\right\}^{\perp}$ are Riemannian, and an isometry $\mathcal{J}: \Gamma^{\perp} \rightarrow \hat{\Gamma}^{\perp}$ such that

$$
\Omega=\left\{(\eta, \mathcal{J} \eta): \eta \in \Gamma^{\perp}\right\} \subset \Gamma^{\perp} \oplus \hat{\Gamma}^{\perp},
$$

with $\hat{\alpha}_{\hat{\Gamma}^{\perp}}=\mathcal{J} \circ \alpha_{\Gamma^{\perp}}^{\prime}$ giving the same identification as before. Note that $\mathcal{J}\left(f^{\prime}\right)=\hat{f}$ and $\mathcal{J}\left(e_{0}\right)=\xi_{0}$.

The preceding ad hoc inclusion in $\Gamma^{\perp}$ and $\hat{\Gamma}^{\perp}$ of the position vectors $f^{\prime}$ and $\hat{f}$, respectively, despite the fact that they are not contained in the subspaces spanned by the images of $\alpha^{\prime}$ and $\hat{\alpha}$, requires a few arguments to 
show that some properties of the bundles used in the proofs in [4], which were automatic in the Riemannian case, still hold in our situation. We will prove these in the form of numbered claims.

Define $\beta$ and $\Theta$ as in the nondegenerate case, but $S \subseteq \Gamma^{\perp}\left(=\hat{\Gamma}^{\perp}\right)$ as

$$
S=\operatorname{span}\left\{f^{\prime}\right\} \oplus \mathcal{S}\left(\left.\alpha^{\prime}\right|_{\Theta \times T M}\right) .
$$

Claim 1. The subbundle $S$ is Lorentzian.

Proof. As in the beginning of the proof of Lemma 13 in [4], we easily check that $\mathcal{S}(\beta)$ is nondegenerate. By $(2.10)$ and Theorem 3 in [6] (see also Corollary 17 in [4]), we have that $\operatorname{dim} \Theta \geq n-\operatorname{dim} \mathcal{S}(\beta) \geq n-p-q>0$. The claim follows from the definition of $S$ and the fact that $f^{\prime} \in S$ is null, since by $(2.11)$,

$$
\left\langle\alpha^{\prime}(Z, Z), f^{\prime}\right\rangle=-\|Z\|^{2} \neq 0, \quad \text { for all } \quad 0 \neq Z \in \Theta .
$$

We also have that $\Theta=\mathcal{N}\left(\alpha_{S^{\perp}}^{\prime}\right) \cap \mathcal{N}\left(\hat{\alpha}_{\hat{S}^{\perp}}\right)$. Then, we define $S_{0}, \mathcal{K}, L^{\ell}, \mathcal{T}$ and $D^{d}$ just as before. Observe that, since $f^{\prime}$ and $\hat{f}$ are normal parallel, we obtain

$$
f^{\prime} \in L^{\ell} \subseteq S_{0}
$$

Claim 2. The subbundle $S_{0} \subseteq S$ is Lorentzian.

Proof. In our setting, the proof of Lemma 12 in [4] implies that the tensor $\mathcal{K}$ as a map $\mathcal{K}: T M \rightarrow \Lambda^{2}(S)$ satisfies that $\operatorname{Im} \mathcal{K}(Z) \subset S \cap \mathcal{S}\left(\left.\alpha^{\prime}\right|_{\Theta \times T M}\right)^{\perp}$, for all $Z \in \Theta$. But since $f^{\prime} \in \operatorname{Ker} \mathcal{K}(Z)=(\operatorname{Im} \mathcal{K}(Z))^{\perp}$, we obtain that $\operatorname{Im} \mathcal{K}(Z)$ $\subset S \cap S^{\perp}=0$, that is,

$$
\mathcal{K}(Z)=0, \quad \text { for all } \quad Z \in \Theta,
$$

and the statement of Lemma 12 in [4] holds here also. But then $\alpha^{\prime}(Z, Z) \in$ $S_{0}$, and the result follows from (2.12) and the fact that $f^{\prime} \in S_{0}$ is null.

By the above two claims and the antisymmetry of $\mathcal{K}$, exactly as in [4] we have the orthogonal splitting

$$
S=S_{0} \oplus^{\perp} S_{1},
$$

with $S_{1}=\operatorname{span}\left\{\mathcal{K}(X) S_{1}: X \in T M\right\}$, which is a Riemannian subbundle. Moreover, by Claim 2 and (2.10) we have that $\operatorname{dim} S_{1} \leq 5$. 
Claim 3. The bilinear map $\gamma=\left.\alpha_{S_{1}}^{\prime}\right|_{\Theta \times T M}$ satisfies $S_{1}=\mathcal{S}(\gamma)$.

Proof. Consider $\xi \in S_{1} \cap \mathcal{S}(\gamma)^{\perp}$. Hence, $\xi \in \mathcal{S}\left(\left.\alpha^{\prime}\right|_{\Theta \times T M}\right)^{\perp}$ since $S_{0} \perp S_{1}$. But then $\xi \perp f^{\prime} \in S_{0}$, and $\xi \in S \cap S^{\perp}=0$.

Claim 4. It holds that $d>0$, and the subbundle $L^{\ell}$ is Lorentzian.

Proof. Using the previous claims, one can argue exactly as in the proof of Theorem 14 in [4] to conclude that

$$
d \geq n-p-q+2 \ell>0
$$

bearing in mind Remark 20 part 3 in [4]. Now, we get from the definition of $D$ and (2.12) applied to $0 \neq Z \in D$ that $f^{\prime} \notin L^{\perp}$. The result now follows from the fact that $f^{\prime} \in L$.

These claims are all we need to make a straightforward check that the proofs of Theorems 11 and 14 in [4] still work in the degenerate case with the preceding definitions. We obtain that, along each connected component of an open dense subset of $M^{n}$, the pair $\left(\mathcal{T}, D^{d}\right)$ for $\left\{f^{\prime}, \hat{f}\right\}$ satisfies $\left(\mathrm{C}_{1}\right)$ and $\left(\mathrm{C}_{2}\right)$ in $(2.1)$, and that the immersions $f^{\prime}$ and $\hat{f}$ have mutually $\Delta^{d+r}$-ruled isometric extensions $F^{\prime}: N^{n+r} \rightarrow \mathbb{L}^{n+p+2}$ and $\hat{F}: N^{n+r} \rightarrow \mathbb{L}^{n+q+2}$. Since $f^{\prime} \in L^{\ell}$, we have that $f^{\prime} \in \Delta^{d+r}=\mathcal{N}(\phi)$ as in (2.2). Thus, by (2.3), both $F^{\prime}(N)$ and $\hat{F}(N)$ are cones, where a subset $\mathcal{C} \subseteq \mathbb{R}^{m}$ being a cone means that $x \in \mathcal{C}$ implies $t x \in \mathcal{C}$ for $t$ close to 1 .

Observe also that the extensions $F^{\prime}$ and $\hat{F}$ are Lorentzian, since $f^{\prime} \in$ $\Delta^{d+r} \subset T N$ and, for $0 \neq Z \in D^{d}$, we get that $\tilde{\nabla}_{Z} Z \in \Delta^{d+r}$ and $\left\langle\tilde{\nabla}_{Z} Z, f^{\prime}\right\rangle=$ $\left\langle\alpha^{\prime}(Z, Z), f^{\prime}\right\rangle=-\|Z\|^{2}<0$. We also conclude that $\Delta^{d+r}$ is strictly larger than $D^{d} \oplus \operatorname{span}\left\{f^{\prime}\right\}$ (and is, in fact, a Lorentzian subbundle), hence Lemma 2.1 implies that $r \geq 2$.

Moreover, by the observation after Definition 2.1, the pair $\left\{F^{\prime}, \hat{F}\right\}$ is nondegenerate. Hence, under the codimension assumption (2.10), we may apply Theorem 2.1 to $\left\{F^{\prime}, \hat{F}\right\}$ to conclude that

$$
d+r \geq n+r-(p+2-r)-(q+2-r)+3(\ell-r)=n-p-q+3 \ell-4 .
$$

Summarizing, we have the following result:

Proposition 2.2. Let $f: M^{n} \rightarrow \mathbb{R}^{n+p}$ and $\hat{f}: M^{n} \rightarrow \mathbb{V}^{n+q+1} \subset \mathbb{L}^{n+q+2}$ form a degenerate isometric pair of immersions, and set $f^{\prime}=\mathcal{I}(f): M^{n} \rightarrow$ $\mathbb{V}^{n+p+1} \subset \mathbb{L}^{n+p+2}$. Assume that $p+q \leq n-1$ and $\min \{p, q\} \leq 5$. Then, along each connected component of an open dense subset of $M^{n}$, the immersions $f^{\prime}$ and $\hat{f}$ have mutually $\Delta^{s}$-ruled isometric Lorentzian conical 
extensions $F^{\prime}: N^{n+r} \rightarrow \mathbb{L}^{n+p+2}$ and $\hat{F}: N^{n+r} \rightarrow \mathbb{L}^{n+q+2}$ such that $\left\langle F^{\prime}, F^{\prime}\right\rangle=\langle\hat{F}, \hat{F}\rangle$, with

$$
s \geq n-p-q+3 \ell-4, \quad 2 \leq r \leq \ell .
$$

Moreover, there exists a vector bundle isometry $T: \mathcal{L}^{l-r} \rightarrow \hat{\mathcal{L}}^{l-r}$ satisfying the conclusions of Proposition 2.1.

Remark 2.2. Theorem 2.1 still holds if the pair is degenerate without the codimension assumption (2.10), but the proof is not completely analogous to the one in [4]. We omit it here since it is not needed for our purposes.

\section{The main result}

The purpose of this section is to prove Theorem 3.1 below, a slightly more general version of Theorem 1.1. It states that a conformal pair in low codimension extends conformally (possibly trivially), with the extensions satisfying additional properties. The idea is to use the results in the previous section to get a certain type of mutually ruled isometric extensions of the isometric light cone representatives of the conformal immersions. Then, we show how these yield the desired conformal extensions.

We start with the following preliminary fact.

Lemma 3.1. Let $f: M^{n} \rightarrow \mathbb{R}^{n+p}$ be a conformal immersion of a Riemannian manifold, and let $f^{\prime}=\mathcal{I}(f): M^{n} \rightarrow \mathbb{V}^{n+p+1} \subset \mathbb{L}^{n+p+2}$ be its isometric light cone representative. If $f^{\prime}$ is $\Delta$-conformally ruled, then the same holds for $f$. Moreover, the following holds:

(i) There exists $\lambda \in C^{\infty}(M)$ such that the conformal factor $\varphi \in C^{\infty}(M)$ relating the metrics $\langle,\rangle_{f}$ and $\langle,\rangle_{f^{\prime}}$ satisfies Hess $\left.\varphi\right|_{\Delta \times \Delta}=\left.\lambda\langle,\rangle_{f^{\prime}}\right|_{\Delta \times \Delta}$;

(ii) The normal components of the mean curvature vector fields $\eta$ and $\eta^{\prime}$ of the leaves of $\Delta$ for $f$ and $f^{\prime}$ are related by

$$
\eta^{\prime}=\varphi^{-1}\left(d \Psi(\eta)-\varphi \xi+\lambda f^{\prime}\right)
$$

where $\xi=\varphi^{-1} e_{0}-d(\Psi \circ f) \operatorname{grad} \varphi$;

(iii) The symmetric bilinear forms $\beta^{f}=\alpha^{f}-\langle,\rangle_{f} \eta$ and $\beta^{f^{\prime}}=\alpha^{f^{\prime}}-\langle,\rangle_{f^{\prime}}$ $\eta^{\prime}$ are related by

$$
\beta^{f^{\prime}}=\varphi d \Psi\left(\beta^{f}\right)+\varphi^{-1}\left(\operatorname{Hess} \varphi(,)-\lambda\langle,\rangle_{f^{\prime}}\right) f^{\prime},
$$

where the Hessian and the gradient are computed with respect to $\langle,\rangle_{f^{\prime}}$. 
Proof. By Lemma 4 in [15], the second fundamental forms of $f$ and $f^{\prime}$ are related by

$$
\alpha^{f^{\prime}}(,)=d \Psi\left(\varphi \alpha^{f}(,)\right)-\langle,\rangle_{f^{\prime}} \xi+\varphi^{-1} \operatorname{Hess} \varphi(,) f^{\prime} .
$$

Since $f^{\prime}$ is $\Delta$-conformally ruled, there exists a normal vector field $\eta^{\prime} \in T_{f^{\prime}}^{\perp} M$ such that $\left.\alpha^{f^{\prime}}\right|_{\Delta \times \Delta}=\left.\langle,\rangle_{f^{\prime}}\right|_{\Delta \times \Delta} \eta^{\prime}$. It follows that there exists a normal vector field $\eta \in T_{f}^{\perp} M$ and $\lambda \in C^{\infty}(M)$ such that $\left.\alpha^{f}\right|_{\Delta \times \Delta}=\left.\langle,\rangle_{f}\right|_{\Delta \times \Delta} \eta$ and Hess $\left.\varphi\right|_{\Delta \times \Delta}=\left.\lambda\langle,\rangle_{f^{\prime}}\right|_{\Delta \times \Delta}$. Replacing into (3.3) yields (3.1) and (3.2).

Theorem 3.1. Let $f: M^{n} \rightarrow \mathbb{R}^{n+p}$ and $\bar{f}: M^{n} \rightarrow \mathbb{R}^{n+q}$ form a conformal pair, with $p+q \leq n-3$ and $\min \{p, q\} \leq 5$. Then (locally on an open dense subset of $M^{n}$ ) the pair $\{f, \bar{f}\}$ extends conformally (possibly trivially) to a mutually $\Delta^{s}$-conformally ruled pair of immersions $F: N^{n+r} \rightarrow \mathbb{R}^{n+p}$ and $\bar{F}: N^{n+r} \rightarrow \mathbb{R}^{n+q}$, with

$$
\Delta^{s}=\mathcal{N}\left(\beta_{\mathcal{L}^{\perp}}^{F}\right) \cap \mathcal{N}\left(\beta_{\overline{\mathcal{L}}^{\perp}}^{\bar{F}_{1}}\right) \quad \text { and } \quad s \geq n-p-q+3\left(\ell^{\mathrm{c}}+r\right),
$$

where $\mathcal{L}:=L_{\Delta}^{\mathrm{c}}(F), \overline{\mathcal{L}}:=L_{\Delta}^{\mathrm{c}}(\bar{F})$ and $\ell^{\mathrm{c}}:=\operatorname{rank} \mathcal{L}=\operatorname{rank} \overline{\mathcal{L}}$. Moreover, there exists a parallel vector bundle isometry $\mathcal{T}: \mathcal{L} \rightarrow \overline{\mathcal{L}}$ such that $\mathcal{T} \circ \beta_{\mathcal{L}}^{F}=\varphi \beta_{\overline{\mathcal{L}}}^{\bar{F}}$, where $\varphi$ is the conformal factor relating the metrics induced by $\bar{F}$ and $F$.

Proof. Set $\hat{f}=\mathcal{I}(\bar{f}): M^{n} \rightarrow \mathbb{V}^{n+q+1} \subset \mathbb{L}^{n+q+2}$, so that $\{f, \hat{f}\}$ becomes an isometric pair. We consider separately the two possible cases:

(i) The pair $\{f, \hat{f}\}$ is nondegenerate.

In this case, Theorem 2.1 applies and yields maximal isometric $\Delta_{0}^{s_{0}}$ ruled extensions $F^{\prime}: N_{0}^{n+r_{0}} \rightarrow \mathbb{R}^{n+p}$ and $\hat{F}: N_{0}^{n+r_{0}} \rightarrow \mathbb{L}^{n+q+2}$, which form a nondegenerate pair and satisfy the conclusions of Proposition 2.1. Since $\hat{f}$ takes values in the light cone and $M^{n}$ is Riemannian, $\hat{f}$ cannot be ruled, and thus $r_{0} \geq 1$. In particular, we have $\ell=\ell_{0}+r_{0} \geq 1$, and hence (2.9) gives

$$
s_{0} \geq n-p-q-2+3\left(\ell_{0}+r_{0}\right),
$$

where $\ell_{0}$ is the rank of the subbundle $L$ given by (2.8) for the pair $\left\{F^{\prime}, \hat{F}\right\}$, which we denote by $\mathcal{L}_{0}$. Moreover, $\hat{F}$ is transversal to the light cone, for $N_{0}^{n+r_{0}}$ is Riemannian and $\hat{F}$ is ruled. By restricting to an open subset if necessary, we may assume that $\hat{F}$ is an embedding, and hence $N:=$ $\hat{F}^{-1}\left(\hat{F}\left(N_{0}\right) \cap \mathbb{V}^{n+q+1}\right) \supseteq M^{n}$ is an $\left(n+r_{0}-1\right)$-dimensional manifold.

Set $F=F^{\prime} \circ i$ and $\bar{F}=\mathcal{C}(\hat{F} \circ i): N \rightarrow \mathbb{R}^{n+q}$, where $i: N \rightarrow N_{0}$ is the inclusion map. Then $\{F, \bar{F}\}$ is a conformal pair, $F \circ j=f$ and $\bar{F} \circ j=\bar{f}$, where $j$ is the inclusion of $M$ into $N$, and hence $\{F, \bar{F}\}$ is a conformal 
extension of $\{f, \bar{f}\}$. Moreover, $F$ and $\bar{F}$ are mutually $\Delta^{s}$-conformally ruled, where $s=s_{0}-1$ and $\Delta^{s}$ is the distribution on $M$ defined by $d \hat{F}(\Delta)=$ $d \hat{F}\left(\Delta_{0}\right) \cap T \mathbb{V}^{n+q+1}$. Therefore,

$$
s \geq n-p-q+3\left(\ell_{0}+r\right)
$$

with $r=r_{0}-1$, and hence the estimate on $s$ will follow once we prove that $\ell_{0} \geq \ell^{\mathrm{c}}$.

First observe that $\hat{\mathcal{L}}:=L_{\Delta}^{\mathrm{c}}(\hat{F} \circ i) \subset \hat{\mathcal{L}}_{0} \oplus \operatorname{span}\{d \hat{F}(\eta)\}$, where $\eta(x) \in$ $T_{x} N_{0} \cap T_{x}^{\perp} N$ is the normal component of the mean curvature vector at $x \in N$ of the leaf of $\Delta^{s}$ through $x$. On the other hand, we obtain from (3.2) that $d \Psi(\overline{\mathcal{L}}) \subset \hat{\mathcal{L}} \oplus \operatorname{span}\{\hat{F} \circ i\}$, hence

$$
d \Psi(\overline{\mathcal{L}}) \subset \hat{\mathcal{L}}_{0} \oplus \operatorname{span}\{d \hat{F}(\eta)\} \oplus \operatorname{span}\{\hat{F} \circ i\} .
$$

Now, for a unit vector $Z \in \Delta$, we have $\alpha_{\hat{F} \circ i}(Z, Z)=d \hat{F}(\eta)$. So $\langle d \hat{F}(\eta)$, $\hat{F} \circ i\rangle=-1$, since $\left\langle\alpha_{\hat{F} \circ i}(),, \hat{F} \circ i\right\rangle=-\langle$,$\rangle . It follows that the subspace V$ on the right-hand side of (3.4) is Lorentzian. Therefore, the subspace $d \Psi(\overline{\mathcal{L}})$ is a Riemannian subspace of $V$ that is orthogonal to the null vector $\hat{F}$ 。 $i \in V$, hence $d \Psi(\overline{\mathcal{L}})$ has codimension at least two in $V$. We conclude that $\operatorname{rank} \overline{\mathcal{L}} \leq \ell_{0}$, as we wished.

We show now that

$$
\mathcal{T}\left(\beta^{F}(Z, X)\right)=\varphi \beta^{\bar{F}}(Z, X), \quad \text { for } Z \in \Delta, X \in T N
$$

defines a parallel vector bundle isometry $\mathcal{T}: \mathcal{L} \rightarrow \overline{\mathcal{L}}$ such that $\mathcal{T} \circ \beta_{\mathcal{L}}^{F}=$ $\varphi \beta \overline{\overline{\mathcal{L}}}$.

Extend $T_{0}$ to a vector bundle map $T_{1}$ between $\mathcal{L}_{1}:=\mathcal{L}_{0} \oplus \operatorname{span}\left\{\eta_{F}\right\} \subset$ $T_{F}^{\perp} N$ and $\hat{\mathcal{L}}_{1}:=\hat{\mathcal{L}}_{0} \oplus \operatorname{span}\left\{\eta_{\hat{F} \circ i}\right\} \subset T_{\hat{F} \circ i}^{\perp} N$ by setting $\left.T_{1}\right|_{\mathcal{L}_{0}}=T_{0}$ and $T_{1}\left(\eta_{F}\right)$ $=\eta_{\hat{F} \circ i}$. Since $\eta_{F}=d F^{\prime}(\eta)$ and $\eta_{\hat{F} \circ i}=d \hat{F}(\eta)$ belong to $T N_{0}$, it is easily seen that $T_{1}$ is also a parallel vector bundle isometry with $\Delta=\mathcal{N}\left(\alpha_{\mathcal{L}_{1}^{\perp}}^{F}\right) \cap$ $\mathcal{N}\left(\alpha_{\hat{\mathcal{L}}_{1}^{\perp}}^{\hat{F} \circ i}\right)$ and $\alpha_{\hat{\mathcal{L}}_{1}}^{\hat{F}_{0} i}=T_{1} \circ \alpha_{\mathcal{L}_{1}}^{F}$. Moreover, since $\mathcal{L} \subset \mathcal{L}_{1}$, the restriction $T=$ $\left.T_{1}\right|_{\mathcal{L}}: \mathcal{L} \rightarrow \hat{\mathcal{L}}$ defines a parallel vector bundle isometry such that $\beta_{\hat{\mathcal{L}}}^{\hat{F} \circ i}=$ $T \circ \beta_{\mathcal{L}}^{F}$

By (3.2) we have

$$
P \circ \beta^{\hat{F} \circ i}=d \Psi\left(\varphi \beta^{\bar{F}}\right),
$$

where $P: T_{\hat{F} \circ i} \mathbb{L}^{n+q+2} \rightarrow d \Psi\left(T_{\bar{F}} \mathbb{R}^{n+q}\right)$ denotes the orthogonal projection. This implies, in particular, that $\mathcal{T}$ is well defined, and that $\Delta=\mathcal{N}\left(\beta_{\mathcal{L}^{\perp}}^{F}\right) \cap$ 
$\mathcal{N}\left(\beta_{\overline{\mathcal{L}}^{\perp}}^{\bar{F}}\right)$. In addition,

$$
d \Psi \circ \mathcal{T}=P \circ T
$$

which implies by (3.2) that $\mathcal{T}$ is a vector bundle isometry. Moreover,

$$
d \Psi \circ \mathcal{T} \circ \beta_{\mathcal{L}}^{F}=P \circ T \circ \beta_{\mathcal{L}}^{F}=P \circ \beta_{\hat{\mathcal{L}}}^{\hat{F} \circ i}=d \Psi\left(\varphi \beta_{\overline{\mathcal{L}}}^{\overline{\mathcal{L}}}\right),
$$

and therefore $\mathcal{T} \circ \beta_{\mathcal{L}}^{F}=\varphi \beta_{\overline{\mathcal{L}}}^{\overline{\mathcal{F}}}$ as desired.

Finally, we must prove that $\mathcal{T}$ is parallel with respect to the induced connections. For $\xi \in \mathcal{L}$ and $Y \in T N$, we have

$$
\begin{aligned}
d \Psi\left(\mathcal{T}\left(\nabla_{Y}^{\perp} \xi\right)_{\mathcal{L}}\right) & =P\left(T\left(\nabla_{Y}^{\perp} \xi\right)_{\overline{\mathcal{L}}}\right)=P\left(\nabla_{Y}^{\perp} T \xi\right)_{\hat{\mathcal{L}}}=P\left(\tilde{\nabla}_{Y} T \xi\right)_{\hat{\mathcal{L}}}=\left(P \tilde{\nabla}_{Y} T \xi\right)_{P \hat{\mathcal{L}}} \\
& =\left(\tilde{\nabla}_{Y} P T \xi\right)_{P \hat{\mathcal{L}}}=\left(\tilde{\nabla}_{Y} d \Psi \mathcal{T} \xi\right)_{d \Psi \overline{\mathcal{L}}}=\left(d \Psi \tilde{\nabla}_{Y} \mathcal{T} \xi\right)_{d \Psi \overline{\mathcal{L}}} \\
& =d \Psi\left(\tilde{\nabla}_{Y} \mathcal{T} \xi\right)_{\overline{\mathcal{L}}}=d \Psi\left(\nabla_{Y}^{\perp} \mathcal{T} \xi\right)_{\overline{\mathcal{L}}}
\end{aligned}
$$

and the claim follows.

(ii) The pair $\{f, \hat{f}\}$ is degenerate.

By Proposition 2.2, the pair $\left\{f^{\prime}, \hat{f}\right\}$ extends isometrically to mutually $\Delta_{0}^{s_{0}}$-ruled Lorentzian cones $F_{0}^{\prime}: N_{0}^{n+r_{0}} \rightarrow \mathbb{L}^{n+p+2}$ and $\hat{F}_{0}: N_{0}^{n+r_{0}} \rightarrow \mathbb{L}^{n+q+2}$, with

$$
s_{0} \geq n-p-q+3 \ell-4
$$

and $2 \leq r_{0} \leq \ell$. Moreover, we have a parallel vector bundle isometry $T_{0}$ : $\mathcal{L}_{0}^{\ell_{0}} \rightarrow \overline{\mathcal{L}}_{0}^{\ell_{0}}$ that preserves second fundamental forms, with $\ell=\ell_{0}+r_{0}$ and $\Delta_{0}^{s_{0}}=\mathcal{N}\left(\alpha_{\mathcal{L}_{0}^{\perp}}^{F_{0}^{\prime}}\right) \cap \mathcal{N}\left(\alpha_{\hat{\mathcal{L}}_{0}^{\perp}}^{\hat{F}_{0}}\right)$.

Since $f^{\prime}$ is tangent to $F_{0}^{\prime}, e_{0}$ is nowhere normal to $F_{0}^{\prime}$. Thus, $F_{0}^{\prime}$ is transversal to the degenerate hyperplane $\mathcal{H}=\mathcal{H}^{n+p+1}:=\left\{x \in \mathbb{L}^{n+p+2}\right.$ : $\left.\left\langle x, e_{0}\right\rangle=1\right\}$, and we locally define $N_{1}^{n+r_{0}-1}:=F_{0}^{\prime-1}\left(F_{0}^{\prime}\left(N_{0}\right) \cap \mathcal{H}\right) \subset N_{0}^{n+r_{0}}$, $\Delta_{1}^{s_{0}-1}:=\Delta_{0}^{s_{0}} \cap T N_{1}$, and $F_{1}^{\prime}:=\left.F_{0}^{\prime}\right|_{N_{1}}, \hat{F}_{1}:=\left.\hat{F}_{0}\right|_{N_{1}}$.

Now, $F_{1}^{\prime}$ is transversal to $\mathbb{V}^{n+p+1}$, hence we may locally define $N^{n+r}:=$ $F_{1}^{\prime-1}\left(F_{1}^{\prime}\left(N_{1}\right) \cap \mathbb{V}^{n+p+1}\right) \subset N_{1}^{n+r_{0}-1}, \quad \Delta^{s}:=\Delta_{1}^{s_{0}-1} \cap T N$, and $F^{\prime}:=\left.F_{1}^{\prime}\right|_{N}$, $\hat{F}:=\left.\hat{F}_{1}\right|_{N}$, with $s=s_{0}-2, r=r_{0}-2$.

Since $F^{\prime}(N) \subset \mathcal{H} \cap \mathbb{V}^{n+p+1}=\mathbb{E}^{n+p}$, there exists $F: N \rightarrow \mathbb{R}^{n+p}$ such that $F^{\prime}=\Psi \circ F$. On the other hand, using that $\left\langle F_{0}^{\prime}, F_{0}^{\prime}\right\rangle=\left\langle\hat{F}_{0}, \hat{F}_{0}\right\rangle$, it follows that $\hat{F}$ takes values in $\mathbb{V}^{n+q+1}$, and we may define $\bar{F}=\mathcal{C}(\hat{F}): N \rightarrow \mathbb{R}^{n+q}$. Then, as in the nondegenerate case, we obtain that $\{F, \bar{F}\}$ is a conformal pair, $F \circ j=f$ and $\bar{F} \circ j=\bar{f}$, where $j$ is the inclusion of $M$ into $N$, hence $\{F, \bar{F}\}$ is a conformal extension of $\{f, \bar{f}\}$. Moreover, $F$ and $\bar{F}$ are mutually $\Delta^{s}$-conformally ruled. 
The estimate on $s$ also follows as in the nondegenerate case. From (3.6) we have

$$
s=s_{0}-2 \geq n-p-q+3\left(\ell_{0}+r\right),
$$

so it suffices to show that $\ell_{0} \geq \ell^{\mathrm{c}}$. As before, $\mathcal{L}^{\prime}:=L_{\Delta}^{\mathrm{c}}\left(F^{\prime}\right) \subset \mathcal{L}_{0} \oplus$ span $\left\{d F_{1}^{\prime}(\eta)\right\}$, where $\eta(x) \in T_{x} N_{1} \cap T_{x}^{\perp} N$ is the normal component of the mean curvature vector at $x \in N$ of the leaf of $\Delta^{s}$ through $x$. On the other hand, $d \Psi(\mathcal{L}) \subset \mathcal{L}^{\prime} \oplus \operatorname{span}\left\{F^{\prime}\right\}$, hence

$$
d \Psi(\mathcal{L}) \subset \mathcal{L}_{0} \oplus \operatorname{span}\left\{d F_{1}^{\prime}(\eta)\right\} \oplus \operatorname{span}\left\{F^{\prime}\right\}
$$

Arguing as before, we obtain that the subspace $W$ on the right-hand-side of (3.7) is Lorentzian. Therefore, $d \Psi(\mathcal{L})$ is a Riemannian subspace of $W$ that is orthogonal to the null vector $F^{\prime} \in W$, hence it has codimension at least two in $W$.

We claim that (3.5) defines also in this case a parallel vector bundle isometry $\mathcal{T}: \mathcal{L} \rightarrow \overline{\mathcal{L}}$ such that $\mathcal{T} \circ \beta_{\mathcal{L}}^{F}=\varphi \beta_{\overline{\mathcal{L}}}^{\bar{F}}$.

Choose smooth unit vector fields $\xi^{\prime}$ and $\hat{\xi}$ spanning $T_{F_{0}^{\prime}} N_{0} \cap T_{F_{1}^{\prime}}^{\perp} N_{1}$ and $T_{\hat{F}_{0}} N_{0} \cap T_{\hat{F}_{1}}^{\perp} N_{1}$, respectively, and set $\mathcal{L}_{1}:=\mathcal{L}_{0} \oplus \operatorname{span}\left\{\xi^{\prime}\right\} \subset T_{F_{1}}^{\perp} N_{1}$ and $\hat{\mathcal{L}}_{1}:=\hat{\mathcal{L}}_{0} \oplus \operatorname{span}\{\hat{\xi}\} \subset T_{\hat{F}_{1}}^{\perp} N_{1}$. Extend the parallel vector bundle isometry $T_{0}: \mathcal{L}_{0} \rightarrow \hat{\mathcal{L}}_{0}$ to $T_{1}: \mathcal{L}_{1} \rightarrow \hat{\mathcal{L}}_{1}$ by setting $\left.T_{1}\right|_{\mathcal{L}_{0}}=T_{0}$ and $T_{1}\left(\xi^{\prime}\right)=\hat{\xi}$.

Now set $\quad \mathcal{L}_{2}:=\mathcal{L}_{1} \oplus \operatorname{span}\left\{\eta_{F^{\prime}}\right\} \subset T_{F^{\prime}}^{\perp} N$ and $\hat{\mathcal{L}}_{2}:=\hat{\mathcal{L}}_{1} \oplus \operatorname{span}\left\{\eta_{\hat{F}}\right\} \subset$ $T_{\hat{\hat{F}}}^{\perp} N$ and extend $T_{1}$ to $T_{2}: \mathcal{L}_{2} \rightarrow \hat{\mathcal{L}}_{2}$ by setting $\left.T_{2}\right|_{\mathcal{L}_{1}}=T_{1}$ and $T_{2}\left(\eta_{F^{\prime}}\right)=\eta_{\hat{F}}$. Since $\eta_{F^{\prime}}=d F_{1}^{\prime}(\eta)$ and $\eta_{\hat{F}}=d \hat{F}_{1}(\eta)$ belong to $T N_{1}$, it is easily seen that $T_{2}$ is also a parallel vector bundle isometry with $\Delta^{s}=\mathcal{N}\left(\alpha_{\mathcal{L}_{2}^{\perp}}^{F^{\prime}}\right) \cap \mathcal{N}\left(\alpha_{\hat{\mathcal{L}}_{2}^{\perp}}^{\hat{F}}\right)$ and $\alpha_{\hat{\mathcal{L}}_{2}}^{\hat{F}}=T_{2} \circ \alpha_{\mathcal{L}_{2}}^{F^{\prime}}$. Moreover, since $\mathcal{L} \subset \mathcal{L}_{2}$, the restriction $T=\left.T_{2}\right|_{\mathcal{L}}: \mathcal{L} \rightarrow \hat{\mathcal{L}}$ defines a parallel vector bundle isometry such that $\beta_{\hat{\mathcal{L}}}^{\hat{F}}=T \circ \beta_{\mathcal{L}^{\prime}}^{F^{\prime}}$.

By (3.2) we have

$$
P \circ \beta^{\hat{F}}=d \Psi\left(\varphi \beta^{\bar{F}}\right) \text { and } \beta^{F^{\prime}}=d \Psi \circ \beta^{F} .
$$

This implies, in particular, that $\mathcal{T}$ is well defined and that $\Delta=\mathcal{N}\left(\beta_{\mathcal{L}^{\perp}}^{F}\right) \cap$ $\mathcal{N}\left(\beta_{\overline{\mathcal{L}}^{\perp}}\right)$. Moreover,

$$
d \Psi \circ \mathcal{T}=P \circ T \circ d \Psi,
$$

which implies by (3.2) that $\mathcal{T}$ is a vector bundle isometry. Thus,

$$
d \Psi \circ \mathcal{T} \circ \beta_{\mathcal{L}}^{F}=P \circ T \circ d \Psi \circ \beta_{\mathcal{L}}^{F}=P \circ \beta_{\hat{\mathcal{L}}}^{\hat{F}}=d \Psi\left(\varphi \beta_{\overline{\mathcal{L}}}^{\bar{F}}\right)
$$

and the claim is proved. 
The proof that $\mathcal{T}$ is parallel with respect to the induced connections is also analogous to that of the nondegenerate case.

\section{The proofs of Theorem 1.2 and Corollary 1.1}

Proof of Theorem 1.2. If the pair $\{f, \hat{f}\}$ in the proof of Theorem 3.1 is nondegenerate, take the maximal isometric $\Delta^{d+r}$-ruled extensions $F^{\prime}$ : $N^{n+r} \rightarrow \mathbb{R}^{n+p}$ and $\hat{F}: N^{n+r} \rightarrow \mathbb{L}^{n+q+2}, r \geq 1$, of $f$ and $\hat{f}$, respectively, given by Theorem 2.1 .

If $\{f, \hat{f}\}$ is a degenerate pair, let $F_{1}^{\prime}: N_{1}^{n+r} \rightarrow \mathcal{H} \subset \mathbb{L}^{n+p+2}$ and $\hat{F}_{1}: N_{1}^{n+r}$ $\rightarrow \mathbb{L}^{n+q+2}$ be as in the proof of Theorem 3.1 for the degenerate case. Note that $r \geq 1$, since $N_{1}^{n+r}$ has dimension one less than that of the extension $F_{0}^{\prime}: N_{0}^{n+r_{0}} \rightarrow \mathbb{L}^{n+q+2}$ of $f^{\prime}$ as in the same proof, and $r_{0} \geq 2$.

We claim that $F^{\prime}:=\pi \circ F_{1}^{\prime}$ is an immersion, where $\pi: \mathbb{L}^{n+p+2}=\mathbb{L}^{2} \times$ $\mathbb{R}^{n+p} \rightarrow \mathbb{R}^{n+p}$ is the projection onto the second factor. Otherwise, $N_{1}^{n+r}$ is degenerate or, equivalently, $e_{0} \in T_{F_{1}^{\prime}} N_{1} \subset T_{F_{0}^{\prime}} N_{0}=T_{f^{\prime}} M \oplus \Lambda$ (see (2.3)). That is, there is $X_{0} \in T M$ such that $X_{0}+e_{0} \in \Delta_{0}=\mathcal{N}(\phi)$ as in (2.2). By (2.11) and $\mathcal{J}\left(e_{0}\right)=\xi_{0}$, this implies that $\operatorname{Im} d \xi_{0}=\operatorname{Im} \hat{\nabla}^{\perp} \xi_{0} \subseteq L$. On the other hand, $e_{0}$ is also normal to $f^{\prime}$, so $e_{0} \in L \subseteq S_{0}$. It follows that $\xi_{0}$ is constant, since $d \xi_{0}=\hat{\nabla}^{\perp} \xi_{0}=\nabla^{\perp} e_{0}=0$.

We conclude that $\hat{f}=\bar{\Psi} \circ f^{*}$ for some isometric immersion $f^{*}: M^{n} \rightarrow$ $\mathbb{R}^{n+q}$, where $\bar{\Psi}: \mathbb{R}^{n+q} \rightarrow \mathbb{V}^{n+q+1} \subset \mathbb{L}^{n+q+2}$ is the isometric embedding defined as in (1.2) with respect to a pseudo-orthonormal basis of $\mathbb{L}^{n+q+2}$ containing $\xi_{0}$. Since $\bar{\Psi}=T \circ \Psi$ for some orthogonal linear transformation $T$ of $\mathbb{L}^{n+q+2}$, it follows that $\mathcal{I}(\bar{f})=\hat{f}=\bar{\Psi} \circ f^{*}=T \circ \Psi \circ f^{*}=T \circ \mathcal{I}\left(f^{*}\right)$, that is, $\mathcal{I}(\bar{f})$ and $\mathcal{I}\left(f^{*}\right)$ are isometrically congruent in $\mathbb{L}^{n+q+2}$. Therefore $\bar{f}$ and $f^{*}$ are conformally congruent in $\mathbb{R}^{n+q}$. This contradicts our hypothesis and proves our claim.

Thus, setting $N=N_{1}$, also in the degenerate case we obtain maximal isometric $\Delta^{d+r}$-ruled extensions $F^{\prime}: N^{n+r} \rightarrow \mathbb{R}^{n+p}$ and $\hat{F}=\hat{F}_{1}: N^{n+r} \rightarrow$ $\mathbb{L}^{n+q+2}, r \geq 1$, of $f$ and $\hat{f}$, respectively.

As in the proof of Theorem 3.1, since $\hat{F}$ is transversal to the light cone, by restricting to an open subset, if necessary, we may assume that $\hat{F}$ is an embedding, so that $\bar{N}=\hat{F}^{-1}\left(\hat{F}(N) \cap \mathbb{V}^{n+q+1}\right) \supset M^{n}$ is an $(n+r-1)$ dimensional manifold. As before, setting $F=F^{\prime} \circ i$ and $\bar{F}=\mathcal{C}(\hat{F} \circ i): \bar{N} \rightarrow$ $\mathbb{R}^{n+q}$, where $i: \bar{N} \rightarrow N$ is the inclusion map, we have that $\{F, \bar{F}\}$ is a conformal pair, $F \circ j=f$ and $\bar{F} \circ j=\bar{f}$, where $j$ is the inclusion of $M$ into $\bar{N}$. Thus, $\{F, \bar{F}\}$ is a conformal extension of $\{f, \bar{f}\}$.

Since $\{f, \bar{f}\}$ is a genuine conformal pair, we must have $r=1$, hence $\bar{N}=M, F \circ i=f$ and $\mathcal{C}(\hat{F} \circ i)=\bar{f}$. A similar argument shows that any 
isometric extension of the pair $\left\{F^{\prime}, \hat{F}\right\}$ would give a conformal extension of the pair $\{f, \bar{f}\}$, hence $\left\{F^{\prime}, \hat{F}\right\}$ must be a genuine isometric pair.

Proof of Corollary 1.1. Given a conformal immersion $\bar{f}: M^{n} \rightarrow \mathbb{R}^{n+q}$, Theorem 3.1 applies and yields, locally on an open dense subset of $M^{n}$, a (possibly trivial) conformal extension $\{F, \bar{F}\}$ of $\{f, \bar{f}\}$ to a mutually $\Delta^{s_{-}}$ conformally ruled pair of immersions $F: N^{n+r} \rightarrow \mathbb{R}^{n+p}$ and $\bar{F}: N^{n+r} \rightarrow$ $\mathbb{R}^{n+q}, 0 \leq r \leq p$, with $s \geq n-p-q+3\left(\ell^{\mathrm{c}}+r\right)$. It suffices to prove that $r=p$.

First we show that $L_{\Delta}^{\mathrm{c}}(f)^{\perp}=\{0\}$. Otherwise, if $s^{\prime}:=\operatorname{rank} L_{\Delta}^{\mathrm{c}}(f)^{\perp}>0$, we would have, since $\Delta \subset \mathcal{N}\left(\beta_{L_{\Delta}^{\mathrm{c} \perp}}^{f}\right)$, that

$$
\nu_{s^{\prime}}^{\mathrm{c}} \geq s \geq n-p-q+3\left(p-s^{\prime}\right)=n+p-q-2 s^{\prime}+\left(p-s^{\prime}\right),
$$

contradicting our assumption on $\nu_{s^{\prime}}^{\mathrm{c}}$. Therefore, we have

$$
s \geq n+2 p-q
$$

Now assume that $r<p$. Since $F$ is $\Delta$-conformally ruled, we have that $\alpha_{F}=\langle,\rangle \eta_{F}$ on $\Delta \times \Delta$ for some normal vector field $\eta_{F}$. In particular, for any unit normal vector field $\xi \in T_{F}^{\perp} N$ we obtain that $\left\langle\left(A_{\xi}^{f}-\left\langle\xi, \eta_{F}\right\rangle I\right) D, D\right\rangle=0$, where $D=\Delta \cap T M$. Since $\operatorname{rank} D=s-r \geq n-(q-p)+1$, then $\nu_{1}^{\mathrm{c}} \geq n-$ $2(q-p)+2$, and this is a contradiction with our assumption on $\nu_{1}^{\mathrm{c}}$.

\section{References}

[1] E. Cartan, La déformation des hypersurfaces dans l'espace euclidien réel a $n$ dimensions, Bull. Soc. Math. France 44 (1916), 65-99.

[2] E. Cartan, La déformation des hypersurfaces dans l'espace conforme réel a $n \geq 5$ dimensions. Bull. Soc. Math. France 45 (1917), 57-121.

[3] M. do Carmo and M. Dajczer, Conformal Rigidity, Amer. J. Math. 109 (1987), 963-985.

[4] M. Dajczer and L. Florit, Genuine deformations of submanifolds, Comm. Anal. Geom. 12 (2004), 1105-1129.

[5] M. Dajczer and L. Florit, Genuine rigidity of Euclidean submanifolds in codimension two, Geom. Dedicata 106 (2004), 195-210. 
[6] M. Dajczer and L. Florit, Compositions of isometric immersions in higher codimension, Manuscripta Math. 105 (2001), 507-517. Erratum, Manuscripta Math. 110 (2003), 135.

[7] M. Dajczer and L. Florit, On conformally flat submanifolds, Comm. Anal. Geom. 4 (1996), 261-284.

[8] M. Dajczer and L. Florit, A counterexample to a conjecture on flat bilinear forms, Proc. Amer. Math. Soc. 132 (2004), 3703-3704.

[9] M. Dajczer, L. Florit and R. Tojeiro, On deformable hypersurfaces in space forms, Ann. Mat. Pura Appl. 174 (1998), 361-390.

[10] M. Dajczer and R. Tojeiro, On Cartan's conformally deformable hypersurfaces, Michigan Math. J. 47 (2000), 529-557.

[11] M. Dajczer and R. Tojeiro, A rigidity theorem for conformal immersions, Indiana Univ. Math. J. 46 (1997), 491-504.

[12] M. Dajczer and R. Tojeiro, Conformal deformations of submanifolds in codimension two, J. Math. Soc. Japan 52 (2000), 41-50.

[13] V. Sbrana, Sulla varietá ad $n-1$ dimensioni deformabili nello spazio euclideo ad $n$ dimensioni, Rend. Circ. Mat. Palermo 27 (1909), 1-45.

[14] S. Silva, On isometric and conformal rigidity of submanifolds, Pacific J. Math. 199 (2001), 227-247.

[15] R. Tojeiro, Isothermic submanifolds of Euclidean space, J. Reine Angew. Math. 598 (2006), 1-24.

IMPA - Estrada Dona Castorina

110 22460-320 RIO DE JANEIRO

BRAZIL

E-mail address: luis@impa.br

Universidade Federal de SÃo Carlos

13565-905 SÃo CARLOS

BRAZIL

E-mail address: tojeiro@dm.ufscar.br

Received August 8, 2009

Dedicated to Marcos Dajczer on his 60th birthday 
